\title{
Genetic Study of Three Closely Linked X chromosome STR Markers in an Argentinian Population
}

Keywords: DNA typing; Short tandem repeats; $X$ chromosome; DXS10079; DXS10074; DXS10075

\begin{abstract}
Three closely linked X chromosome markers (DXS10079, DXS10074 and DXS10075) were evaluated in a sample of 213 unrelated individuals (106 women and 107 men) from Buenos Aires (Argentina). Hardy-Weinberg equilibrium (HWE) was tested in the female sample and no significant deviations were observed. Homogeneity of allele frequencies of men and women was compared by Fisher's exact test and showed similar distributions. Overall linkage disequilibrium (LD) tests were performed in males for all pairs of loci and significant associations were detected only in the pair DXS10074-DXS10075. However, significant inter-allelic associations were detected among the three markers when the disequilibrium coefficient ( $\left.D^{\prime}\right)$ was estimated. Comparison of allele frequencies among Argentinian, Asian, European, and Latin Americ an populations was performed and parameters of forensic interest were also estimated.
\end{abstract}

\section{Introduction}

$\mathrm{X}$-chromosome short tandem repeat (X-STR) markers have attracted increasing interest in the forensic community due to their advantage over autosomal and Y chromosome markers in kinship cases where the alleged father is absent and the child is female [1,2]. There are four linkage groups of STR markers distributed along the $\mathrm{X}$ chromosome [3]. Linkage group 2 contains a cluster composed of markers DXS10074, DXS10074 and DXS10075 spanning a 280-kb region at Xq12 that can provide stable haplotypes to solve complex kinship scenarios [4]. The use of closely located linked markers is of special interest for the reconstruction of the genetic structure of lineages $[4,5]$. Although several studies have documented allele frequencies of X-STR markers for different ethnic groups [6-8] further population genetic data is necessary in order to increase the knowledge and applicability of these markers in forensic genetics. The objective of the present study is to perform a population study of three closely linked X-STR markers -DXS10079, DXS10074 and DXS10075 in an Argentinian sample set, to estimate parameters of forensic interest and to compare the allele frequencies with those in Japanese, Chinese, German and Brazilian population sets.

\section{Materials and Methods}

\section{Population}

Blood samples were collected from 213 unrelated individuals from Buenos Aires city (106 women and 107 men) with informed consent. Samples were stripped of any personal identifiers and made anonymous prior to testing at our lab. The protocols used in this study were approved by the Institutional Review Board of Sam Houston State University.

\section{Journal of}

Forensic Investigation

\section{Pablo Noseda ${ }^{1}$, Michael Hernandez ${ }^{2}$, Brittney Gonzalez ${ }^{2}$, Sheree Hughes-Stamm ${ }^{2}$ and David Gangitano ${ }^{2 *}$}

${ }^{1}$ Laboratorio de Huellas Digitales Geneticas. Secretaria Nacional de Niñez, Adolescencia y Familia (SENAF). Paz Soldan 5200, Buenos Aires, Argentina C1427DSJ

${ }^{2}$ Department of Forensic Science, College of Criminal Justice, Sam Houston State University, 1003 Bowers Blvd., Huntsville, Texas 77340, USA

\section{Address for Correspondence}

David Gangitano, Ph.D., Department of Forensic Science, College of Criminal Justice, Sam Houston State University, 1003 Bowers Blvd., Office 221C, Huntsville, Texas 77340, USA, Tel: +1 936294 4413; fax: +1 936-294-4905; E-mail : dag006@shsu.edu

Submission: 23 September 2013

Accepted: 22 October 2013

Published: 24 October 2013

\section{DNA extraction and quantification}

Genomic DNA (gDNA) was extracted from blood samples using a modified Chelex ${ }^{\circledR}$ method [9] suspending the resin in TE buffer (Tris-HCl 10mM, EDTA 0.1 mM, pH 8.0). DNA samples were quantified by real time PCR in a StepOne ${ }^{\mathrm{TM}}$ Real Time System (Life technologies, Carlsbard, CA) using $2 \mu \mathrm{L}$ extracted gDNA, 400nM D21S11 primers (GenBank Accession number AP000433) (Integrated DNA Technologies, Coralville, IA), and 1X SYBR ${ }^{\circledR}$ Green PCR Master Mix (Life technologies). The following real time PCR cycling parameters were used: $10 \mathrm{~min}$ at $95^{\circ} \mathrm{C}$ and 40 cycles of $15 \mathrm{~s}$ at $95^{\circ} \mathrm{C}$ and $1 \mathrm{~min}$ at $60^{\circ} \mathrm{C}$.

\section{PCR amplification and genotyping}

Three X-STRs were amplified in a multiplex amplification reaction including DXS10079, DXS10074 and DXS10075 according to previous studies [4]. Amplified PCR products were detected by capillary electrophoresis in either an ABI PRISM ${ }^{\mathrm{TM}} 310$ or a 3500 Genetic Analyzer (Life Technologies). Allele calling was performed using the software GeneMapper v. 4.1 (Life Technologies) and compared with allelic ladders as recommended by the ISFG [10]. A custom designed bin set was implemented to allow automation of genotyping. A ladder containing all the observed alleles in the population was prepared according to Sajantila et al. [11]. Allelic ladders were designed for each X STR marker using a mixture of individual samples that represent each allele observed in the population data set. The samples were amplified separately for each locus accordingly to the previously described protocol. Each of the amplified products were diluted 1:1,000 with $\mathrm{H}_{2} \mathrm{O}$, mixed together in a total volume of $1 \mathrm{~mL}$ and re-amplified under the same conditions but using 20 cycles instead of 30 . The ladder was calibrated with DNA control samples 9947A, 9948, and K562 (Promega, Madison, WI) s [12]. Microvariants 14.3 (DXS10074), 17.2, 18.2, and 19.2 (DXS10075) were sequenced. DXS10074 and DXS10075 primers were used in a direct Taq-cycle sequencing procedure using the BigDye ${ }^{\circledR}$ Direct Cycle Sequencing Kit and the 3500 Genetic Analyzer with Sequencing 
Citation: Noseda P, Hernandez M, Gonzalez B, Hughes-Stamm S, Gangitano D. Genetic Study of Three Closely Linked X chromosome STR Markers in an Argentinian Population. J Forensic Investigation. 2013;1(2): 4.

Analysis software version 4.1 (Life Technologies). Sequences were assembled and proof-read using the Geneious Pro software v. 5.5.7 (Biomatters Ltd., Auckland, New Zealand). Sequenced alleles were used to calibrate the allelic ladder. Allele frequencies and parameters of forensic interest were generated with PowerStats v. 1.2 software [13]. Hardy-Weinberg equilibrium (HWE) in females, observed heterozygosity and gene diversity were carried out with the Genetic Data Analysis software [14]. Homogeneity of allele frequencies of men and women and overall linkage disequilibrium (LD) in the male sample was assessed using GENEPOP version 4.1 software package [15]. Inter-allelic linkage disequilibrium (ID) in males was estimated with the MIDAS software [16]. Power of exclusion calculations were estimated according to Desmarais et al. [17] Population comparisons were carried out by estimating genetic distances based on Fstusing the Arlequin v. 3.5 software [18]. Bonferroni correction for the number of loci analyzed in HWE and LD evaluation and the correction for the number of inter-population comparisons at adjusted significance level were performed [19].

\section{Quality control}

The laboratory of the first author successfully participated in proficiency testing of the Latin-American Society of Forensic Genetics 2007 (http://www.slagf.org.ar).

\section{Results and Discussion}

Combined allele frequencies for men and women and parameters of forensic interest are depicted in Table 1. Fisher's exact test did not reveal allele distribution differences between men and women, therefore their frequencies can be combined. No significant deviations from expectations of HWE were detected in any of the markers analyzed in women ( $p>0.015$; significant level after Bonferroni correction). Overall LD and ID are shown in Table 2. Analysis of the three X-STR markers in males showed no detectable evidence of LD except for pair DXS10074-DXS10075 ( $<<0.015$; significant level after Bonferroni correction). These results are consistent with the close genetic distance of these two markers $(21 \mathrm{~kb})$. Moreover, significant inter-allelic associations were detected among these three markers when the disequilibrium coefficient (D') was evaluated (D'> 0.8) (Table 2). Most studies in forensic X-STRs use pvalues of a Fisher's exact test as a measured variable to infer the value of gametic disequilibrium (GD); which is not a test to evaluate GD. In linkage studies, it is important not only to measure overall linkage disequilibrium between pairs of loci but also to detect specific interallelic associations between markers, which can be evaluated using D' [20]. A value of D'> 0.8 indicates the presence of strong linkage disequilibrium between two markers [21]. Haplotype frequencies for males are displayed in Table 3.

Microvariants observed in loci DXS10074 and DXS10075 were sequenced and submitted to Genbank (Accession numbers JX645356, JX645357, JX645358 and JX645359). Interestingly, the repeat structure of allele 14.3 in DXS10074 reflects the motif of "short" alleles $(<10)$ instead of "long" alleles ( $>13)$, since it is composed of contiguous repetitions of the (AAGA) $)_{n}$ unit, with a AGA downstream the core repeat unit [4]. The repeat structure of microvariants $17.2,18.2$, and 19.2 of marker DXS10075 is consistent with the repeat pattern of intermediate X.2 alleles described by Hering et al. [4].

Population sample comparisons by pairwise genetic distance analysis, based on Fst were performed with previously published
Table 1: Allele frequencies and parameters of forensic interest for three linked X-STR markers in an Argentinian population (106 women and 107 men; 319chromosomes tested).

\begin{tabular}{|c|c|c|c|}
\hline Alleles & DXS10079 & DXS10074 & DXS10075 \\
\hline 7 & - & 0.0392 & - \\
\hline 8 & - & 0.0945 & - \\
\hline 9 & - & 0.0323 & - \\
\hline 12 & - & 0.0046 & - \\
\hline 13 & 0.0047 & 0.0138 & 0.0386 \\
\hline 14 & 0.0419 & 0.0276 & 0.0091 \\
\hline 14.3 & - & 0.0023 & - \\
\hline 15 & 0.0465 & 0.0991 & 0.0273 \\
\hline 16 & 0.0465 & 0.1636 & 0.2523 \\
\hline 16.2 & - & - & 0.0023 \\
\hline 17 & 0.0372 & 0.2627 & 0.375 \\
\hline 17.2 & - & - & 0.0864 \\
\hline 18 & 0.1465 & 0.1866 & 0.175 \\
\hline 18.2 & - & - & 0.0091 \\
\hline 19 & 0.1977 & 0.0645 & 0.0182 \\
\hline 19.2 & - & - & 0.0068 \\
\hline 20 & 0.2442 & 0.0069 & - \\
\hline 21 & 0.1488 & 0.0023 & - \\
\hline 22 & 0.0605 & - & - \\
\hline 23 & 0.0186 & - & - \\
\hline 24 & 0.007 & - & - \\
\hline $\mathrm{He}_{\mathrm{f}}$ & 0.8558 & 0.8426 & 0.737 \\
\hline $\mathrm{Ho}_{\mathrm{f}}$ & 0.8679 & 0.8113 & 0.7 \\
\hline$H W_{E} f$ & 0.596 & 0.541 & 0.296 \\
\hline PIC & 0.8354 & 0.8199 & 0.6932 \\
\hline$P D_{f}$ & 0.9511 & 0.9509 & 0.8922 \\
\hline$P D_{m}$ & 0.8361 & 0.8423 & 0.7737 \\
\hline$P E_{\text {trio }}$ & 0.8285 & 0.8251 & 0.7196 \\
\hline$P E_{\text {motherless }}$ & 0.7216 & 0.717 & 0.5846 \\
\hline
\end{tabular}

$\mathrm{He}_{\mathrm{f}}$ : gene diversity in females, $\mathrm{Ho}_{\mathrm{f}}$ : observed heterozygosity in females, $\mathrm{HWE}_{\mathrm{f}}$ $p$-values of Hardy- Weinberg equilibrium test in females, PIC: polymorphic information content, $P D_{f}$ : power of discrimination in females, $P D$ : power of discrimination in males. PE: power of exclusion in parentage testing involving a daughter.

Table 2: Overall linkage disequilibrium (LD) and inter-allelic linkage disequilibrium (D') among three X-STR markers in Argentinean males ( $N=107)$.

\begin{tabular}{|c|c|c|c|c|}
\hline Marker 1 & Marker 2 & LD $^{\mathbf{a}}$ & Allele 1_Allele 2 & D $^{\text {'b }}$ \\
\hline DXS10079 & DXS10074 & 0.086 & $19 \_7$ & 1.0000 \\
\hline DXS10079 & DXS10075 & 0.178 & $15 \_17$ & 1.0000 \\
\hline & & & $24 \_17$ & 1.0000 \\
\hline & & & $19 \_18.2$ & 1.0000 \\
\hline & & & $18 \_19.2$ & 1.0000 \\
\hline DXS10074 & DXS10075 & $0.000^{c}$ & $7 \_13$ & 0.8237 \\
\hline & & & $17 \_18.2$ & 1.0000 \\
\hline & & & $17 \_19.2$ & 1.0000 \\
\hline & & & $7 \_13$ & 1.0000 \\
\hline
\end{tabular}

a p-value of linkage disequilibrium exact test (3200 shufflings)

' D': disequilibrium coefficient. Reported only observed D' values $>0.8$

c Statistically significant difference at $p<0.015$ (after Bonferroni correction).

data that contained the cluster DXS10079-DXS10074-DXS10075, when available. These included Brazil [22], China [23], Japan [24],and Germany [4] (Table 4). Results showed statistically 
Citation: Noseda P, Hernandez M, Gonzalez B, Hughes-Stamm S, Gangitano D. Genetic Study of Three Closely Linked X chromosome STR Markers in an Argentinian Population. J Forensic Investigation. 2013;1(2): 4.

ISSN: 2330-0396

significant differences between all population pairs except for Alagoas-Rio, Buenos Aires-Germany and when the comparison involved the Argentinian and Brazilian population samples. This is not unexpected because the three South American populations (Buenos Aires, Alagoas and Rio) were subjected to a similar Amerindian and European (Portuguese for Brazil and Spaniard/

Table 3: Haplotype frequencies of three X-STR markers in males from Buenos Aires population $(\mathrm{N}=107)$

\begin{tabular}{|c|c|c|c|c|}
\hline DXS10079 & DXS10074 & DXS10075 & number & frequency \\
\hline 20 & 7 & 13 & 2 & 0.01869 \\
\hline 18 & 8 & 16 & 3 & 0.02804 \\
\hline 19 & 8 & 17 & 3 & 0.02804 \\
\hline 15 & 17 & 17 & 3 & 0.02804 \\
\hline 19 & 17 & 17 & 3 & 0.02804 \\
\hline 19 & 17 & 18 & 3 & 0.02804 \\
\hline 18 & 7 & 13 & 2 & 0.01869 \\
\hline 18 & 16 & 16 & 2 & 0.01869 \\
\hline 19 & 16 & 17 & 2 & 0.01869 \\
\hline 20 & 15 & 17 & 2 & 0.01869 \\
\hline 20 & 16 & 16 & 2 & 0.01869 \\
\hline 21 & 17 & 16 & 2 & 0.01869 \\
\hline 20 & 18 & 17 & 2 & 0.01869 \\
\hline 19 & 18 & 18 & 2 & 0.01869 \\
\hline 20 & 17 & 18 & 2 & 0.01869 \\
\hline 20 & 18 & 17.2 & 2 & 0.01869 \\
\hline 21 & 18 & 17 & 2 & 0.01869 \\
\hline 21 & 18 & 17.2 & 2 & 0.01869 \\
\hline 20 & 19 & 17.2 & 2 & 0.01869 \\
\hline 22 & 17 & 18 & 2 & 0.01869 \\
\hline 19 & 7 & 13 & 1 & 0.00935 \\
\hline 20 & 7 & 14 & 1 & 0.00935 \\
\hline 17 & 8 & 17 & 1 & 0.00935 \\
\hline 18 & 8 & 17 & 1 & 0.00935 \\
\hline 19 & 8 & 16 & 1 & 0.00935 \\
\hline 18 & 8 & 18 & 1 & 0.00935 \\
\hline 21 & 8 & 16 & 1 & 0.00935 \\
\hline 19 & 8 & 18 & 1 & 0.00935 \\
\hline 18 & 9 & 13 & 1 & 0.00935 \\
\hline 17 & 9 & 16 & 1 & 0.00935 \\
\hline 18 & 9 & 17 & 1 & 0.00935 \\
\hline 19 & 9 & 17 & 1 & 0.00935 \\
\hline 18 & 13 & 14 & 1 & 0.00935 \\
\hline 16 & 13 & 17 & 1 & 0.00935 \\
\hline 19 & 14 & 17 & 1 & 0.00935 \\
\hline 21 & 14 & 16 & 1 & 0.00935 \\
\hline 20 & 14 & 18 & 1 & 0.00935 \\
\hline 19 & 15 & 16 & 1 & 0.00935 \\
\hline
\end{tabular}

\begin{tabular}{|c|c|c|c|c|}
\hline 19 & 15 & 17 & 1 & 0.00935 \\
\hline 20 & 15 & 16 & 1 & 0.00935 \\
\hline 21 & 15 & 17 & 1 & 0.00935 \\
\hline 19 & 15 & 19 & 1 & 0.00935 \\
\hline 20 & 15 & 18 & 1 & 0.00935 \\
\hline 14 & 16 & 16 & 1 & 0.00935 \\
\hline 14 & 16 & 17 & 1 & 0.00935 \\
\hline 16 & 16 & 16 & 1 & 0.00935 \\
\hline 17 & 16 & 16 & 1 & 0.00935 \\
\hline 16 & 16 & 17 & 1 & 0.00935 \\
\hline 16 & 16 & 18 & 1 & 0.00935 \\
\hline 20 & 16 & 15 & 1 & 0.00935 \\
\hline 18 & 16 & 17 & 1 & 0.00935 \\
\hline 21 & 16 & 15 & 1 & 0.00935 \\
\hline 20 & 16 & 17 & 1 & 0.00935 \\
\hline 21 & 16 & 17 & 1 & 0.00935 \\
\hline 22 & 16 & 17 & 1 & 0.00935 \\
\hline 23 & 16 & 17 & 1 & 0.00935 \\
\hline 22 & 16 & 18 & 1 & 0.00935 \\
\hline 14 & 17 & 16 & 1 & 0.00935 \\
\hline 17 & 17 & 16 & 1 & 0.00935 \\
\hline 20 & 17 & 16 & 1 & 0.00935 \\
\hline 18 & 17 & 18 & 1 & 0.00935 \\
\hline 18 & 17 & 19.2 & 1 & 0.00935 \\
\hline 19 & 17 & 18.2 & 1 & 0.00935 \\
\hline 21 & 17 & 17 & 1 & 0.00935 \\
\hline 21 & 17 & 17.2 & 1 & 0.00935 \\
\hline 21 & 17 & 18 & 1 & 0.00935 \\
\hline 20 & 17 & 19 & 1 & 0.00935 \\
\hline 22 & 17 & 17.2 & 1 & 0.00935 \\
\hline 24 & 17 & 17 & 1 & 0.00935 \\
\hline 16 & 18 & 15 & 1 & 0.00935 \\
\hline 16 & 18 & 16 & 1 & 0.00935 \\
\hline 15 & 18 & 17 & 1 & 0.00935 \\
\hline 19 & 18 & 16 & 1 & 0.00935 \\
\hline 18 & 18 & 17 & 1 & 0.00935 \\
\hline 19 & 18 & 17 & 1 & 0.00935 \\
\hline 20 & 18 & 16 & 1 & 0.00935 \\
\hline 20 & 18 & 19 & 1 & 0.00935 \\
\hline 23 & 18 & 17.2 & 1 & 0.00935 \\
\hline 14 & 19 & 16 & 1 & 0.00935 \\
\hline 20 & 19 & 17 & 1 & 0.00935 \\
\hline 19 & 19 & 18 & 1 & 0.00935 \\
\hline 20 & 19 & 18 & 1 & 0.00935 \\
\hline
\end{tabular}


Citation: Noseda P, Hernandez M, Gonzalez B, Hughes-Stamm S, Gangitano D. Genetic Study of Three Closely Linked X chromosome STR Markers in an Argentinian Population. J Forensic Investigation. 2013;1(2): 4.

Table 4: Genetic distances based on pairwise comparisons (Fst) and associated p-values (in parentheses) among Buenos Aires, Asian, European and LatinAmerican populations.

\begin{tabular}{|c|c|c|c|c|c|}
\hline & Buenos Aires & Han & Alagoas & Rio & Japan \\
\hline \multirow[t]{2}{*}{ Han $(\text { China })^{20}$} & 0.008 & & & & \\
\hline & $(0.000)^{a}$ & & & & \\
\hline \multirow[t]{2}{*}{ Alagoas $\left(\right.$ Brazil) ${ }^{19}$} & 0.003 & 0.018 & & & \\
\hline & $(0.180)$ & $(0.000)^{a}$ & & & \\
\hline \multirow[t]{2}{*}{ Rio (Brazil) ${ }^{19}$} & 0.002 & 0.019 & 0.000 & & \\
\hline & $(0.225)$ & $(0.000)^{a}$ & $(0.360)$ & & \\
\hline \multirow[t]{2}{*}{ Japan $^{21}$} & 0.018 & 0.009 & 0.022 & 0.025 & \\
\hline & $(0.000)^{a}$ & $(0.000)^{a}$ & $(0.000)^{a}$ & $(0.000)^{a}$ & \\
\hline \multirow[t]{2}{*}{ Germany $^{5}$} & 0.005 & 0.012 & 0.010 & 0.013 & 0.015 \\
\hline & $(0.036)$ & $(0.000)^{\mathrm{a}}$ & $(0.000)^{\mathrm{a}}$ & $(0.000)^{\mathrm{a}}$ & $(0.000)^{a}$ \\
\hline
\end{tabular}

aStatistically significant differences at $p<0.003$ (after Bonferroni correction).

Italian for Argentina) complex admixture process. Although X STR comparative data between Argentina and Germany were not found in the literature, two previous reports showed similarities in allele frequencies between Buenos Aires and other European sample sets when other X STR markers were used (i.e., Spain, Portugal and Italy) $[25,26]$. The genetic differences observed between Japanese and Chinese populationswere previously reported when autosomal single nucleotide polymorphisms were evaluated [27]. In conclusion, a population database for the cluster DXS10079-DXS10074-DXS10075 was developed for forensic and anthropological purposes. The use of these linked markers in conjunction with other X-STR markers should be considered especially in deficiency cases when a daughter is involved and complex kinship scenarios.

\section{References}

1. Gusmao L, Alves C, Gomes I, and Sanchez-Diz P (2012) Capillary electrophoresis of an X-chromosome STR decaplex for kinship deficiency cases. Methods Mol Biol 830: 57-71.

2. Szibor R (2007) X-chromosomal markers: past, present and future. Forensic Sci Int Genet 1: 93-99.

3. Szibor R, Krawczak M, Hering S, Edelmann J, Kuhlisch E, et al. (2003) Use of X-linked markers for forensic purposes. Int J Legal Med 117: 67-74.

4. Hering S, Augustin C, Edelmann J, Heidel M, Dressler J, et al. (2006) DXS10079, DXS10074 and DXS10075 are STRs located within a 280-kb region of $\mathrm{Xq} 12$ and provide stable haplotypes useful for complex kinship cases. Int J Legal Med 120: 337-345.

5. Szibor R, Hering S, Kuhlisch E, Plate I, Demberger S, et al. (2005) Haplotyping of STR cluster DXS6801-DXS6809-DXS6789 on Xq21 provides a powerful tool for kinship testing. Int J Legal Med 119: 363-369.

6. Asamura H, Sakai H, Kobayashi K, Ota M, Fukushima H (2006) MiniX-STR multiplex system population study in Japan and application to degraded DNA analysis. Int J Legal Med 120: 174-181.

7. Hedman M, Palo JU, Sajantila A (2009) X-STR diversity patterns in the Finnish and the Somali population. Forensic Sci Int Genet 3: 173-178.

8. Ribeiro Rodrigues EM, Leite FP, Hutz MH, Palha Tde J, Ribeiro dos Santos AK, et al. (2008) A multiplex PCR for $11 \mathrm{X}$ chromosome STR markers and population data from a Brazilian Amazon Region. Forensic Sci Int Genet 2: 154-158.

9. Walsh PS, Metzger DA, Higuchi R (1991) Chelex 100 as a medium for simple extraction of DNA for PCR-based typing from forensic material. Biotechniques 10: 506-513.

10. Bar W, Brinkmann B, Budowle B, Carracedo A, Gill P, et al. (1997) DNA recommendations. Further report of the DNA Commission of the ISFH regarding the use of short tandem repeat systems. International Society for Forensic Haemogenetics. Int J Legal Med 110: 175-176.

11. Sajantila A, Puomilahti S, Johnsson V, Ehnholm C (1992) Amplification of reproducible allele markers for amplified fragment length polymorphism analysis. Biotechniques 12: 16, 18, 20-22.

12. Szibor R, Edelmann J, Hering S, Plate I, Wittig H, et al. (2003) Cell line DNA typing in forensic genetics--the necessity of reliable standards. Forensic Sci Int 138: 37-43

13. Tereba A, Tools for analysis of population statistics, in Profiles in DNA1999, Promega Corporation.

14. Lewis P, Zaykin D (2001) Genetic Data Analysis: Computer program for the analysis of allelic data. Version 1.0.

15. Rousset $F$ (2008) genepop'007: a complete re-implementation of the genepop software for Windows and Linux. Molecular Ecology Resources 8:103-106.

16. Gaunt TR, Rodriguez S, Zapata C, and Day IN (2006) MIDAS: software for analysis and visualisation of interallelic disequilibrium between multiallelic markers. BMC Bioinformatics 7: 227.

17. Desmarais D, Zhong Y, Chakraborty R, Perreault C, Busque L (1998) Development of a highly polymorphic STR marker for identity testing purposes at the human androgen receptor gene (HUMARA). J Forensic Sci 43: $1046-1049$

18. Excoffier L, Laval G, Schneider S (2005) Arlequin (version 3.0): an integrated software package for population genetics data analysis. Evol Bioinform Online 1: 47-50.

19. Evett W, Weir BS (1998) Interpreting DNA evidence. Statistical genetics for forensic scientists. Sinauer Associates Inc, Sunderland, MA: 209.

20. Medina-Acosta E (2011) Evidence of partial and weak gametic disequilibrium across clusters of pericentromeric short tandem repeats loci on human $X$ chromosome: Proceed with caution in forensic genetics. Forensic Sci In Genet 5: 545-547.

21. Oki T, Hayashi T, Ota M, Asamura H (2012) Development of a multiplex assay with 16 SNPs on X chromosome for degraded samples. Leg Med 14: 11-16.

22. Ferreira da Silva IH, Barbosa AG, Azevedo DA, Sanchez-Diz P, Gusmao L, et al. (2010) An X-chromosome pentaplex in two linkage groups: haplotype data in Alagoas and Rio de Janeiro populations from Brazil. Forensic Sci Int Genet 4: e95-100.

23. Liu QL, Lu DJ, Quan L, Chen YF, Shen M, et al. (2012) Development of multiplex PCR system with 15 X-STR loci and genetic analysis in three nationality populations from China. Electrophoresis 33: 1299-1305.

24. Samejima M, Nakamura Y, Minaguchi K (2011) Population genetic study of six closely linked groups of X-STRs in a Japanese population. Int J Legal Med 125: 895-900.

25. Bobillo C, Sala A, Gusmao L, Corach D (2011) Genetic analysis of 10 X-STRs in Argentinian population. Forensic Sci Int Genet 5: e14-e16.

26. Noseda PA, Kenline J, Manning S, Gangitano DA (2013) Population data for DXS6800, DXS101 and DXS8377 loci from Buenos Aires (Argentina). J Forensic Leg Med 20: 522-524.

27. Abdulla MA, Ahmed I, Assawamakin A, Bhak J, Brahmachari SK, et al. (2009) Mapping human genetic diversity in Asia. Science 326: 1541-1545. 